\title{
Purification and characterization of circulating Onchocerca volvulus antigens from epileptic and non-epileptic onchocerciasis patient sera
}

\author{
Christopher B. TUME ${ }^{1 *}$, Christian Delaunay Tchokote FATCHEU ${ }^{1}$, \\ Edwige Rosine TIODJIO ${ }^{1}$, Mireille DONGMO ${ }^{1}$, Gilbert ATEUFACK ${ }^{2}$, \\ Donatien GATSING ${ }^{1}$, Andre Pagnah ZOLI ${ }^{3,4}$ and Tazoacha ASONGANYI ${ }^{5}$ \\ ${ }^{I}$ Department of Biochemistry, Faculty of Science, University of Dschang, P.O. Box 67 Dschang, Cameroon. \\ ${ }^{2}$ Department of Animal Biology, Faculty of Science, University of Dschang, P.O. Box 67 Dschang, Cameroon. \\ ${ }^{3}$ Department of Animal Productions, Faculty of Agronomy and Agricultural Sciences (F.A.S.A), University of \\ Dschang, P.O. Box 222 Dschang, Cameroon. \\ ${ }^{4}$ School of Veterinary Medicine and Sciences, University of Ngaoundéré, P.O. Box 454 Ngaoundéré, \\ Cameroon. \\ ${ }^{5}$ Department of Biochemistry, Faculty of Medicine and Biomedical Sciences, University of Yaoundé I, \\ Cameroon. \\ *Corresponding author, E-mail: tumechrist@yahoo.com, Tel: +237 77578688 / +2379908 1771
}

\begin{abstract}
Studies conducted during the past 25 years to investigate the possible relationship between onchocerciasis and epilepsy have led to contradictory results. In the present study aimed at contributing to the investigation of a possible relationship between onchocerciasis and epilepsy, we proceeded to purify and characterize circulating $O$. volvulus antigens from sera of onchocerciasis patients with and without epilepsy. Out of 539 onchocerciasis patients included in the study, sera from 78 epileptics and 20 non epileptics with high antigen titres were separately pooled and subjected to affinity purification using immunosorbent columns prepared using human and rabbit anti-O. volvulus $\operatorname{IgG}$ antibodies. Eluates of purified circulating $O$. volvulus antigens were concentrated, and then the protein contents were determined using the Bradford method. The antigenicity of the purified antigens was evaluated in a direct ELISA using onchocerciasis patient sera. Finally, the molecular composition of the purified proteins was determined by SDS-PAGE. The purified antigens were highly antigenic and there was no significant difference in the reaction profiles of the two groups or categories of patients. SDS-PAGE analysis showed that the purified antigens ranged from 31.63 to $102.40 \mathrm{KDa}$ and there was no difference in the molecular composition of antigens purified from sera of the two classes of patients. Based on this antigen profiling between epileptic and non-epileptic onchocerciasis patients, we cannot conclude with certainty whether onchocerciasis is really a cause of epilepsy in areas where it is hyperendemic as predicted by some epidemiological studies.

(C) 2012 International Formulae Group. All rights reserved.
\end{abstract}

Keywords: Antigen-detection ELISA, Immunoadsorbent columns, Affinity chromatography, Antigenicity, SDS-PAGE.

\section{INTRODUCTION}

Onchocerciasis or "river blindness" is a parasitic disease caused by the filaral worm Onchocerca volvulus, and transmitted by the bite of a blackfly, Simulium. It causes serious cutaneous lesions and can involve in its final phase, an irreversible blindness. It is estimated that about some 40 million people in the world currently suffer from the disease, among which the majority are in tropical Africa (Pion et al., 2009). Studies carried out in several African countries indicate, that 
onchocerciasis hyperendemic zones also have a high prevalence of epilepsies (World Health Organization, 1999).

Epilepsy is an affection due to an abnormal, sudden and excessive electric discharge which comes from an injured area of the brain, called the electric focus. This cerebral attack is a type of neurological disease comparable with an electric storm occurring in the brain, and which can be more or less violent, localised and frequent (Tecoma and Corey-Bloom, 2009). According to Pion et al. (2009), epilepsy is particularly common in tropical areas and one main reason for this is that many endemic infections have neurological consequences. There are no geographical, social or racial boundaries to the disease and everyone can suffer from it. In addition, the medical, social and demographic burden of epilepsy remain substantial in tropical countries where it is often seen as a contagious condition and where the aetiology is often undetermined. The causes of the disease are multiple, including those due to infections affecting the brain such as the presence of Taenia solium metacestodes in the brain, leading to neurocysticercosis which is one of the major causes of epilepsy in areas where cysticercosis is endemic (De Bittencourt et al., 1996; Carpio et al., 1998).

For several decades, field researchers have reported some overlapping between the geographical distribution of epilepsy and onchocerciasis. To this effect, the question of the existence of a causal relationship between onchocerciasis and epilepsy has been the subject of many studies, essentially based on epidemiologic surveys. However, the results obtained up to date are controversial and many unclarified points persist. The kind of relationship that exists between these two diseases, as well as the epileptogenic nature of $O$. volvulus, need to be explained. We undertook the present study to purify and characterise $O$. volvulus antigens from epileptic and non-epileptic onchocerciasis patients, so as to certify whether or not the antigen profiles differ in the two groups of onchocerciasis patients, as this may give us a clue as to the role of onchocerciasis in the development of epilepsy in areas where the disease is hyperendemic.

\section{MATERIALS AND METHODS Study subjects}

Subjects included in this study comprised epileptics and non-epileptics from the onchocerciasis endemic localities of Batibo-Widikum in the North-West region and Bangoua in the West region of Cameroon. After an information campaign by medical authorities only those from whom we had informed consent were included in the study. Patient identification was done on the basis of clinical signs of the two diseases. It is necessary to note here that an interview was carried out for the purpose of obtaining information on the age of the patient, age at which epileptic seizures began, their manifestations, their frequency, and possibly the responsible factors. A total of 539 onchocerciasis subjects comprising 387 epileptics from Batibo-Widikum and 54 from Bangoua, as well as 98 non-epileptics from Bangoua were included in the study.

\section{Blood collection and sera preparation}

Five millilitres $(5 \mathrm{ml})$ of blood were collected in dry centrifuge tubes by venopuncture at the level of the arm. The blood was transported to the laboratory and allowed to clot overnight at $+5{ }^{\circ} \mathrm{C}$ in a refrigerator, then centrifuged at $1500 \mathrm{rpm}$ for 15 minutes and the supernatant (serum) collected and stored at $-20{ }^{\circ} \mathrm{C}$ until use.

Negative control sera for this study consisted of sera from 65 European children who had never been exposed to onchocerciasis and showed no symptoms or signs of epilepsy.

\section{Identification of O.volvulus seropositive patient sera by antigen detection ELISA (sandwich-ELISA)}

The Sandwich-ELISA technique was essentially carried out as previously described (Tume et al., 1997), using O. volvulus-specific anti-Oncho-C27 IgG antibodies, with all incubations done at $37{ }^{\circ} \mathrm{C}$ on an orbital shaker. The anti-Oncho-C27 $\mathrm{IgG}$, was diluted to a final concentration of $1 \mu \mathrm{g} / \mathrm{ml}$ in $0.1 \mathrm{M}$ Carbonate/Bicarbonate buffer $\mathrm{pH}$ 9.6, and 100 $\mu 1$ dispensed into each well of microtitre plates, and incubated for 60 minutes at $37^{\circ} \mathrm{C}$. Plates were emptied of their contents, washed 
with Phosphate-buffered saline (PBS), $\mathrm{pH} 7.2$, containing $0.05 \%$ Tween-20 (PBS-T), and blocked by incubating for 45 minutes at $37{ }^{\circ} \mathrm{C}$ with $150 \mu \mathrm{l} /$ well of blocking buffer $(0.01 \%$ New Born calf serum in PBS-T). The contents of each plate were again discarded and replaced with $100 \mu \mathrm{l} /$ well of patient (test) or control sera. Plates were incubated for 60 minutes at $37{ }^{\circ} \mathrm{C}$, then emptied and washed thoroughly as above. One hundred microlitres $(100 \mu \mathrm{l})$ per well of biotinylated anti-OnchoC27 IgG antibodies diluted 1000-fold in blocking buffer were introduced, and then the plates were incubated for 60 minutes at $37^{\circ} \mathrm{C}$. They were emptied and washed thoroughly again as above. One hundred microlitres (100 $\mu 1)$ of peroxidase-conjugated streptavidin diluted 3000-fold in blocking buffer were then dispensed per well, and incubated for 30 minutes at $37{ }^{\circ} \mathrm{C}$. The plates were once again thoroughly washed and further incubated in the dark at room temperature with $100 \mu \mathrm{l} /$ well of peroxidase substrate (O-phenylene diamine), prepared according to the manufacturer (Sigma). The incubation lasted for about 20 minutes during which time a significant colour change of the substrate occurred in serpositive wells. Reactions were stopped by adding $25 \mu \mathrm{l} /$ well of $4 \mathrm{M}$ Sulphuric acid $\left(\mathrm{H}_{2} \mathrm{SO}_{4}\right)$, and the absorbance values determined spectrophotometrically at $492 \mathrm{~nm}$ using an automated ELISA plate reader.

All the above incubation steps, except that of the substrate were carried out at $37{ }^{\circ} \mathrm{C}$ with gentle shaking on a rocking platform.

The cut-off value was established as the mean plus 2 times the standard deviation of the absorbance values for the 65 negative control sera $\left(\mathrm{X}_{\mathrm{Neg}}+2 \mathrm{SD}\right)$.

Seropositive sera from epileptics were pooled into one lot and those from nonepileptics into another lot, then labelled and stored at $-20^{\circ} \mathrm{C}$.

Purification of $O$. volvulus circulating antigens by affinity chromatography

The immunoadsorbent columns were prepared using the inert matrix Sephacryl S100 gel, onto which human anti-O. volvulus $\mathrm{IgG}$ on the one hand, and rabbit anti- $O$. volvulus $\operatorname{IgG}$ antibodies on the other, were coupled. The activation and the coupling were carried out as previously described (Sanderson and Wilson, 1971; Wilson and Nakane, 1976; Tume et al., 1997). Purification of the circulating $O$. volvulus antigens was made from the onchocerciasis-epileptic and onchocerciasis-non-epileptic sera using the two types of immunoaffinity columns. In each case, $10 \mathrm{ml}$ of the pooled serum were thawed and divided into two equal volumes $(5 \mathrm{ml}$ each), one for purification through the human anti-O. volvulus IgG column and the other through the rabbit anti-O. volvulus IgG column. The affinity chromatography was done as previously described (Ngu et al., 1989). The bound $O$. volvulus antigens were eluted using acetate buffer (1.5M Acetic acid, $0.5 \mathrm{M} \mathrm{NaCl}, \mathrm{pH} 2.5$ ). The protein-containing eluates were pooled and dialysed against 2 litres of PBS with two buffer changes, and then concentrated using sephadex G25 powder.

The protein concentration of each antigen preparation was determined using the Bradford method (1976), and the samples were aliquoted and stored frozen at $-20{ }^{\circ} \mathrm{C}$ for subsequent characterisation .

\section{Characterization of circulating $O$. volvulus antigens \\ Comparative antigenicity of purified $O$. volvulus antigens \\ The antibody detection ELISA} technique used was essentially carried out as previously described (Tume et al., 1997), with some modifications. The purified antigen, in each case was diluted to a final concentration of $1 \mu \mathrm{g} / \mathrm{ml}$ in $0.1 \mathrm{M}$ Carbonate/Bicarbonate buffer $\mathrm{pH} 9.6$, and $100 \mu \mathrm{l}$ dispensed into each well of microtitre plates, and incubated for 45 minutes at $37{ }^{\circ} \mathrm{C}$. Plates were emptied, washed with Tris-buffered saline (pH 7.2) containing $0.05 \%$ Tween 20 (TBS-T), and blocked by incubating for 45 minutes at $37{ }^{\circ} \mathrm{C}$ with $150 \mu \mathrm{l} /$ well of blocking buffer $(0.01 \%$ New Born calf serum in TBS-T). The contents of each plate were discarded again and replaced with $100 \mu \mathrm{l} /$ well of either control sera, onchocerciasis-epileptic sera or onchocerciasis-non epileptic sera, each diluted 1000-fold in blocking buffer above. Plates were incubated for 60 minutes at $37{ }^{\circ} \mathrm{C}$, then emptied and washed thoroughly as above. One 
hundred microlitres $(100 \mu \mathrm{l})$ of peroxidaseconjugated goat anti-human IgG antibodies diluted 3000-fold in blocking buffer was then dispensed per well, and incubated for 15 minutes at $37{ }^{\circ} \mathrm{C}$. The plates were once again thoroughly washed and incubated at room temperature with $100 \mu \mathrm{l} /$ well of peroxidase substrate (O-phenylene diamine), prepared according to the manufacturer (Sigma). After colour development, the reactions were stopped by adding $25 \mu \mathrm{l} /$ well of $4 \mathrm{M}$ Sulphuric acid $\left(\mathrm{H}_{2} \mathrm{SO}_{4}\right)$ and the absorbances read at 492 $n m$ using an ELISA plate reader.

All the above incubation steps, except that of the substrate were carried out with gentle shaking on a rocking platform.

\section{SDS-PAGE analysis of purified antigens}

This electrophoretic analysis was done as previously described (Towbin and Gopdon, 1979; Tume, 1993). Briefly, a 15\% reducing acrylamide gel was introduced into an electrophoresis tank containing the migration buffer (25 mM Tris/192 mM glycine/0.1\% SDS). Four volumes of purified antigens from the two patient categories and the two types of affinity columns were each mixed with one volume of $5 \mathrm{X}$ concentrated Gel Loading Buffer (GLB) then heated for 5 minutes at $95{ }^{\circ} \mathrm{C}$. The samples were loaded into the gel wells alongside the Molecular Weight protein markers (Fermentas) and the electrophoresis carried out for 2 hours at 90 $\mathrm{mA}$. The gel was then removed and stained with Coomassie Brillant blue R-250 and then destained as previously described (Towbin and Gopdon, 1979 and Tume, 1993).

The relative mobilities of the Molecular Weight standards and the protein bands for each purified antigen sample were then calculated. A standard curve of the relative mobilities versus $\log _{10}$ of molecular weight of standards was drawn and used to determine the corresponding molecular weight of each purified protein band.

\section{Statistical analysis}

The data obtained were analyzed using the software SPSS 10.0 for Windows, and the data expressed in the form of Mean \pm Standard Deviation. All the statistical analyses were done using the Student $t$ test to the threshold of $5 \%$.

\section{RESULTS \\ Detection of circulating $O$. volvulus antigens in patient sera}

Table 1 presents the seroprevalence of $O$. volvulus antigens in the study subjects per locality. Seropositivity to $O$. volvulus antigens was defined relative to a threshold or cut-off value, here defined as the mean absorbance of negative controls, plus 2 times the standard deviation $\left(\mathrm{X}_{\mathrm{Neg}}+2 \mathrm{SD}\right)$. Based on this, all test subjects whose optical densities are greater than this threshold of detection are declared $O$. volvulus seropositive and all those with the optical density below the threshold are declared seronegative.

According to Table 1, $19.12 \%$ of sera from epileptics from Batibo-Widikum tested positive for circulating $O$. volvulus antigens, whereas in Bangoua with a mixed population of epileptics and non epileptics, only $7.4 \%$ of epileptics were seropositive. Globally, of the 539 onchocerciasis patients comprising 441 epileptics and 98 non-epileptics included in the study, $78(17.7 \%)$ of the epileptics and 20 $(20.4 \%)$ of the non-epileptics were seropositive for $O$. volvulus antigens, giving a total prevalence of circulating $O$. volvulus antigens in the study population of $18.2 \%$. No significant difference $(\mathrm{P}>0.05)$ in seroprevalence was observed between epileptics (17.7\%) and non epileptics (20.4\%). Also, the mean absorbances characteristic of the antigen levels in the two study populations did not show any significant difference $(\mathrm{P}>0.05)$.

\section{Characterization of circulating $O$. volvulus antigens}

Antigenicity of purified $O$. volvulus antigens

Table 2 presents the antigenicity of purified $O$. volvulus antigens with sera from onchocerciasis patients presenting with or without epilepsy. Antigenicity of $O$. volvulus antigens was defined relative to a threshold or cut-off value, here defined as the mean absorbance of negative controls, plus 3 times the standard deviation $\left(\mathrm{X}_{\mathrm{Neg}}+3 \mathrm{SD}\right)$. 
Table 1: Frequency of seropositivity for $O$. volvulus antigens according to epilepsy status.

\begin{tabular}{|c|c|c|c|c|c|c|c|c|c|}
\hline \multirow[t]{2}{*}{ Village } & \multicolumn{2}{|c|}{$\begin{array}{c}\text { Number of } \\
\text { subjects and } \\
\text { status } \\
\end{array}$} & \multicolumn{2}{|c|}{$\begin{array}{c}\text { Number of } \\
\text { positives per } \\
\text { status } \\
\end{array}$} & \multicolumn{2}{|c|}{$\begin{array}{l}\text { Percentage of } \\
\text { positives per } \\
\text { status }(\%)\end{array}$} & \multicolumn{2}{|c|}{$\begin{array}{c}\text { Mean of optical density }(\mathrm{X} \pm \mathrm{SD}) \text { per } \\
\text { status }\end{array}$} & \multirow[t]{2}{*}{$\begin{array}{r}\text { Cut-off value } \\
\left(\mathrm{X}_{\mathrm{Neg}}+2 \mathrm{SD}\right)\end{array}$} \\
\hline & $\mathbf{E}^{+}$ & $\mathbf{E}^{-}$ & $\mathbf{E}^{+}$ & $\mathbf{E}^{-}$ & $\mathbf{E}^{+}$ & $\mathbf{E}^{-}$ & $\mathbf{E}^{+}$ & $\mathbf{E}^{-}$ & \\
\hline Bangoua & 54 & 98 & 04 & 20 & 7.4 & 20.4 & $0.545 \pm 0.013$ & $0.590 \pm 0.085$ & NA \\
\hline sub-totals & 441 & 98 & 78 & 20 & 17,7 & 20.4 & I & I & NA \\
\hline TOTAL & \multicolumn{2}{|c|}{539} & \multicolumn{2}{|c|}{98} & \multicolumn{2}{|c|}{18,2} & \multicolumn{2}{|c|}{ I } & NA \\
\hline
\end{tabular}

$\mathrm{E}^{+}$: Epileptics; E: Non epileptics.

$\mathrm{X}_{\mathrm{Neg}}+$ 2SD: Mean absorbance of negative controls plus 2 times the Standard Deviation.

Table 2: Antigenicity of purified $O$. volvulus antigens with sera from onchocerciasis patients with different epilepsy status.

\begin{tabular}{|c|c|c|c|c|c|c|c|c|c|}
\hline \multirow[t]{2}{*}{ Village } & \multicolumn{2}{|c|}{$\begin{array}{c}\text { Number of } \\
\text { subjects and } \\
\text { status } \\
\end{array}$} & \multicolumn{2}{|c|}{$\begin{array}{c}\text { Number of } \\
\text { positives per } \\
\text { status } \\
\end{array}$} & \multicolumn{2}{|c|}{$\begin{array}{c}\text { Percentage of } \\
\text { positives by } \\
\text { status }(\%) \\
\end{array}$} & \multicolumn{2}{|c|}{$\begin{array}{l}\text { Mean of optical density }(\mathrm{X} \pm \mathrm{SD}) \text { per } \\
\text { status }\end{array}$} & \multirow[t]{2}{*}{$\begin{array}{l}\text { Cut-off value } \\
\left(\mathrm{X}_{\mathrm{Neg}}+3 \mathrm{3SD}\right)\end{array}$} \\
\hline & $\mathbf{E}^{+}$ & $\mathbf{E}^{-}$ & $\mathbf{E}^{+}$ & $\mathbf{E}^{-}$ & $\mathbf{E}^{+}$ & $\mathbf{E}^{-}$ & $\mathbf{E}^{+}$ & $\mathbf{E}^{-}$ & \\
\hline Bangoua & 50 & 55 & 50 & 55 & 100 & 100 & $1.450 \pm 0.849$ & $1.381 \pm 0.843$ & NA \\
\hline sub-totals & 250 & 55 & 250 & 55 & 100 & 100 & l & l & NA \\
\hline TOTAL & \multicolumn{2}{|c|}{305} & \multicolumn{2}{|c|}{305} & \multicolumn{2}{|c|}{100} & \multicolumn{2}{|c|}{ 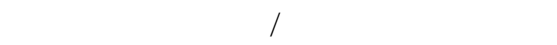 } & NA \\
\hline
\end{tabular}

$\mathrm{E}^{+}$: Epileptics; E: Non epileptics.

$\mathrm{X}_{\mathrm{Neg}}+$ 3SD: Mean absorbance of negative controls plus 3 times the Standard Deviation. 
Table 2 shows that, $100 \%$ of epileptics from Batibo-Widikum presented with circulating $O$. volvulus antibodies in their sera. In Bangoua with a mixed population of epileptics and non epileptics, $100 \%$ of epileptics were seropositive for circulating $O$. volvulus antibodies. Globally, $100 \%$ of epileptics in the entire study population have been, exposed at least once, to $O$. volvulus worms during their lifetime.

\section{SDS-PAGE}

Electrophoretic separation of the various protein components of the antigens immunosorbed from sera of the two patient categories showed that the purified $O$. volvulus antigens were composed of proteins ranging in molecular weight from 31.63 to $102.40 \mathrm{KDa}$, and there was no difference in the protein profiles of circulating $O$. volvulus antigens purified from the two patient categories and using the two immunosorbent columns (results not shown).

\section{DISCUSSION}

The aim of this study was to contribute to the understanding of the relationship between onchocerciasis and epilepsy by evaluating the profile of antigens purified from epileptic and non-epileptic onchocerciasis patient sera, as well as the antigenicity of these purified antigens vis a vis onchocerciasis patient sera from epileptics and non epileptics.

Our results showed no significant difference in the levels of seroprevalence of $O$. volvulus antigens between the sera of epileptic and non-epileptic onchocerciasis patients. These findings correlate with those of Kaiser et al. (2011) in which they found no significant difference in the skin microfilarial load of epilepsy patients compared to the nonepileptic controls. Indeed, studies of Kabore et al. (1996), Druet-Cabanac et al. (1999) and Farnarier et al. (2000) undertaken in different geographical areas that are both hyperendemic for onchocerciaisis and with high epilepsy prevalences did not conclude that there is a relationship between the two diseases.
However, other studies including those of Ovuga et al. (1992), Kipp et al. (1994), Kaiser et al. (1996) and Newell et al. (1997) supported the existence of such a relationship. In addition to these conflicting findings, we do not have any information on the exact chronological order of occurrence of the two diseases among the study populations. In fact, if onchocerciasis was the first disease affecting these patients, its implication as the aetiological agent of epilepsy could be ascribed. On the other hand, if epilepsy was the first disease to affect these patients, then the possible role of onchocerciasis in epilepsies could be highlighted by an increase in the severity of seizures. The existence of no significant difference in the levels of seroprevalence between the two groups of patients $(17.7 \%$ prevalence among epileptics and $20.4 \%$ among non-epileptics) suggests that there may exist no correlation between their onchocercal infection and the development of epilepsy. Although in a study using magnetic resonance imagery (MRI) and cerebrospinal fluid examination (Kaiser et al., 2000), no $O$. volvulus parasites were found in the brains of epileptics from an onchocerciasis endemic area, it is likely that antigenic components rather than the intact parasites may be at the origin of epilepsy in some patients.

In a recent review, Wagner and Newton (2009) raised the interesting hypothesis that immunological responses to helminths including $O$. volvulus, might be involved in the pathogenesis of epilepsy. This correlates very well with findings from previous studies which had shown that immunological mechanisms including the production of some cytokines are implicated in the occurrence of certain epilepsies (Newton and Forster, 1996). Moreover, it was equally shown that cytokines play a role not only in epilepsies (Aarli et al., 2003) but also in onchocerciasis (Ward et al., 1988; King and Nutman, 1991; Soboslay et al., 1992; Chan et al., 1993). Therefore, the implication of onchocerciasis in occurrence of epilepsies could not only be of direct nature through the 
various ways by which circulating $O$. volvulus antigens or $O$. volvulus microfilariae would induce a hyperexcitability of a more or less extended neuron population (Molyneux et al., 1989; Newton and Foster, 1996), but also of indirect nature through the immunological mechanisms involving IgE (Buttner et al., 1982) and cytokine production ((Molyneux et al., 1989; Finkelman et al., 1991; Modlin and Nutman, 1993; Newton and Foster, 1996).

Our study also showed a very low prevalence of $O$. volvulus antigens within the epileptic sera. This low prevalence rate suggests that either the epilepsy in seronegative epileptics may have a different aetiological origin or that onchocerciasis may not be a cause of epilepsy. Given that one major occupation of people in the BatiboWidikum area is pig rearing, it is likely that neurocysticercosis could be at the origin of their epilepsy; alternatively, their epilepsy could be of a mixed aetiology arising from combined infection with $O$. volvulus and Taenia solium. However, even though the connection between onchocerciasis and epilepsy may be influenced by the presence of other endemic diseases known to be a cause of epilepsy especially cysticercosis (Kaiser et al., 2010; Katabarwa et al., 2008), a study by Kaiser et al. (2008) in areas where a relationship between onchocerciasis and epilepsy had been found, do not support the hypothesis that neurocysticercosis could be the cause of onchocerciasis-associated epileptic seizures.

We also observed no difference in the banding pattern of purified antigens from epileptic and non-epileptic sera. This observation shows that there may exist no difference in the antigens found in the two groups of patients, and thus suggests that if there exists a difference in the molecular composition of the $O$ volvulus antigens circulating in the sera of the two groups of patients, this difference might not be at the macromolecular level but rather at the level of the antigenic epitopes constituting the antigen molecules.
This study thus showed that there is no significant difference in antigen levels circulating in the sera of epileptics and nonepileptics, and also that there is no difference in the molecular composition of these antigens. Also, a very low prevalence of $O$. volvulus circulating antigens was observed among the sera of epileptic patients.

On the basis of the $O$. volvulus antigen profiling between epileptic and nonepileptic onchocerciasis patients obtained in this study, we cannot conclude with certainty whether onchocerciasis is really a cause of epilepsy in areas where it is hyperendemic as predicted by epidemiological studies. Further research is thus needed for a better understanding of the neurological pathogenicity in onchocerciasis.

\section{ACKNOWLEDGEMENTS}

The authors acknowledge with thanks, Dr Nsame Denis, the principal physician of the Batibo District Health Centre, and all the research participants from the villages of Bangoua, Batibo and Widikum for their cooperation.

This work was financially supported by a research subvention from the University of Dschang.

\section{REFERENCES}

Aarli JA. 2003. Role of cytokines in neurological disorders. Current Medicinal Chemistry, 10: 1931-1937.

Bradford MM. 1976. A rapid and sensitive method for the quantification of microgram quantities of proteins utilizing the principle of protein-dye binding. Analytical Biochemistry, 72: 248-254.

Buck AA, Anderson RI, MacRae AA. 1973. Serum immunoglobulin levels in five villages of the republic of Tchad and in onchocerciasis patients with and without microfilariae. Tropical Medicine and Parasitology, 24: 21-31.

Buttner DW, Laer GV, Manweller E, Buttner M. 1982. Clinical, parasitological and serological studies of onchocercisasis in 
the Yemen Arab Republic. Tropical Medicine and Parasitology, 33: 201-212.

Carpio A, Escobar A, Hauser A. 1998. Cysticercosis and epilepsy: A Critical Review. Epilepsia, 39(10): 1025-1040.

Chan C, Li Q, Brezin AP, Whitcup SM, Ewuagu C, Ottessen E, Nussenbaltt RB. 1993. Immunopathology of ocular onchocerciasis. 3. TH-2 helper T-cell in conjunctiva. Ocular Immunology and Inflammation, 1: 71-77.

De Bittencourt PRM, Ademolekum B, Bharucha N, Carpio A, Cossio OH, Danesi MA, Dumas ML, Meinardi H, Ordinario A, Senanayake N, Shakir BS, Sotelo J. 1996. Epilepsy in the tropics: I. Epidemiology, Socioeconomic Risk Factors and Etiology. Epilepsia, 37(11): 1121-1127.

Druet-cabanac M, Preux PM, Bouteille B, Bernardy P, Dunand J, Hopkins A, Yaga G, Tabo A, Sartoris C, Macharia W, Dumas M. 1999. Onchocerciasis and Epilepsy: a matched case-control study in the Central African Republic. American Journal of Epidemiology, 149: 565-570.

Druet-Cabanac M, Boussinesq M, Dongmo L, Farnarier G, Bouteille B, Preux PM. 2004. Review of epidemiological studies searching for a relationship between onchocerciasis and epilepsy. Neuroepidemiology, 23: 144149.

Farnarier G, Diop S, Coulibaly B, Arborio S, Dabo A, Diakité M, Traoré S, Banou A, Nimaga K, Vaz T, Doumbo O. 2000. Onchocercose et épilepsie: Enquête epidemiologique au Mali. Tropical Medicine, 60: 151-155.

Finkelman FD, Pearce EJ, Yrban JF, Shef A. 1991. Regulation and biological functions of helminth-induced cytokine responses. Immunoparasitology Today, 1: 62-66.

Kabore J, Cabore JW, Melaku Z, DruetCabanac M, Preux PM. 1996. Epilepsy in a focus of onchocerciasis in Burkina Faso. Lancet, 347. 836.

Kaiser C, Asaba G, Mugisa C, Kabagambe G, Rating D, Leichsenring M. 1996. The prevalence of epilepsy follows the distribution of onchocerciasis in a West Uganda focus. Bulletin of the World Health Organization, 74: 361-367.

Kaiser C, Benninger C, Asaba G, Mugisa C, Kabagambe G, Kipp W, Rating D. 2000. Clinical and electro-clinical classification of epileptic seizures in West Uganda. Bulletin de la Société de Pathologie Exotique, 93: 255-259.

Kaiser C, Pion S, Preux PM, Kipp W, Dozie I, Boussinesq M. 2008. Onchocerciasis, cysticercosis, and epilepsy. American Journal of Tropical Medicine and Hygiene, 79: 643-644.

Kaiser C, Pion S, Boussinesq M. 2010. Do helminths cause epilepsy? The case of Onchocerca volvulus. Parasite Immunology, 32: 79-80.

Kaiser C, Rubaale T, Tukesiga E, Kipp W, Kabagambe G, Ojony JO, Asaba G. 2011. Association between onchocerciasis and epilepsy in the Itwara hyperendemic focus, West Uganda: Controlling for time and intensity of exposure. American Journal of Tropical Medicine and Hygiene, 85(2): 225-228.

Katabarwa M, Lakwo T, Habumogisha P, Richards F, Eberhard M. 2008. Could neurocysticercosis be the cause of "onchocerciasis-associated" epileptic seizures? American Journal of Tropical Medicine and Hygiene, 78: 400-401.

King CL, Nutman TB. 1991. Regulation of the immune response in lymphatic filariasis and onchocerciasis. Immunoparasitology Today, 1: 54-58.

Kipp W, Kasoro S, Burnham G. 1994. Onchocerchiasis and epilepsy in Uganda. Lancet. 343(8890): 183-184.

Modlin RL, Nutman TB. 1993. Type 2cytokines and negative immune regulation in human infection. Current Opinions in Immunology, 5: 511-517.

Molyneux ME, Taylor TE, Wirima JJ. 1989. Clinical features and prognostic indicators in pediatry cerebral malaria: a study of 131 comatose Malawian children. Quarterly Journal of Medicine, 71: 441-459. 
Newell ED, Vyungimana F, Bradley JE. 1997. Epilepsy, retarded growth and onchocerchiasis in two areas of onchocerchiasis in Burundi. Transactions of the Royal Society of Tropical Medicine and Hygiene, 91(5): 525-527.

Newton CR, Forster D. 1996. Epileptic seizures and malaria in Kenyan children. Transactions of the Royal Society of Tropical Medicine and Hygiene, 9: 152155.

Ngu JL, Tume C, Lando G, Ndumbe P, Leke RGF, Titanji V, Asonganyi T. 1989. Comparative studies of clinical groups of patients in an onchocerciasis endemic area for evidence of immune-mediated protection. Tropical Medicine and Parasitology, 40: 460-463.

Ovuga E, Kipp W, Mungherera M, Kasoro S. 1992. Epilepsy and retarded growth in a hyperendemic focus of onchocerciasis in rural Western Uganda, East African Medical Journal, 69: 554-556.

Pion SDS, Kaiser C, Boutros-Toni F, Cournil A, Taylor MM, Meredith SEO, Stufe A, Bertocchi I, Kipp W, Preux PM, Boussinesq M. 2009. Epilepsy in onchocerciasis endemic areas: systematic review and meta-analysis of populationbased surveys. PLoS Neglected Tropical Diseases, 3: e461.

Sanderson CJ, Wilson DV. 1971. A simple method for coupling proteins to insoluble polysaccharides. Immunology, 20: 10611065.

Soboslay PT, Newland HS, White AT. 1992. Ivermectin effect on microfilariae of Onchocerca volvulus after a single oral dose in humans. Tropical Medicine and Parasitology, 38: 8-10.

Tecoma ES, Corey-Bloom J. 2009. Epilepsies. In Clinical Adult Neurology ( $3^{\text {rd }}$ Edition),
Corey-Bloom J, Ronald D (Eds). Demos Medical Publishing LIC: New York; 229256.

Towbin SHT, Gopdon J. 1979. Electrophoresis transfer of proteins from polyacrylamide gels to nitrocellulose sheets: Procedure and some applications. Proceeding of the National Academy of Science USA, 76: 4350-4354.

Tume CB, Ngu J, McKerrow J, Sun E, Barr P, Bathurst I, Morgan G, Nkenfou C, Asonganyi T, Lando G. 1997. Characterization of a recombinant Onchocerca volvulus antigen (OV33) produced in yeast. The American Journal of Tropical Medicine and Hygiene, 57: 626-633.

Tume CB. 1993. Protective immunity in human onchocerciasis: Identification of operative mechanisms. Doctorat de 3eme Cycle Thesis, University of Yaoundé I, Yaoundé. p135.

Wagner RG, Newton CR. 2009. Do helminths cause epilepsy? Parasite Immunology, 31: 697-705.

Ward DJ, Nutman TB, Zea-Flores G, Portocarrera C, Lujan A, Ottesen EA. 1988. Onchocerciasis and immnity in humans: Enhanced T-cell responsiveness to parasite antigens in putatively immune individuals. The Journal of Infectious Diseases, 157: 536-546.

World Health Organization. 1999. Onchocerciasis and its Control. WHO Technical Report Series No. 852. World Health Organization: Geneva.

Wilson MB, Nakane PK. 1976. The covalent coupling of proteins to periodate-oxidized sephadex: a new approach to immunoadsorbent preparation. Journal of Immunological Methods, 12: 171-181. 